\title{
Technical Note: The horizontal scale dependence of the cloud overlap parameter $\alpha$
}

\author{
I. Astin ${ }^{1}$ and L. Di Girolamo ${ }^{2}$ \\ ${ }^{1}$ Department of Electronic and Electrical Engineering, University of Bath, Bath, BA27AY, UK \\ ${ }^{2}$ Department of Atmospheric Sciences, University of Illinois at Urbana-Champaign, Urbana, IL, USA \\ Correspondence to: I. Astin (i.astin@bath.ac.uk)
}

Received: 10 March 2014 - Published in Atmos. Chem. Phys. Discuss.: 15 April 2014

Revised: 18 July 2014 - Accepted: 22 August 2014 - Published: 19 September 2014

\begin{abstract}
The cloud overlap parameter $\alpha$ relates the combined cloud fraction between two altitude levels in a grid box to the cloud fraction as derived under the maximum and random overlap assumptions. In a number of published studies in this and other journals, it is found that $\alpha$ tends to increase with an increasing scale. In this Technical Note, we investigate this analytically by considering what happens to $\alpha$ when two grid boxes are merged to give a grid box with twice the area. Assuming that $\alpha$ depends only on scale, then between any two fixed altitudes, there will be a linear relationship between the values of $\alpha$ on the two scales. We illustrate this by finding the relationship when cloud cover fractions are assumed to be uniformly distributed, but with varying degrees of horizontal and vertical correlation. Based on this, we conclude that $\alpha$ increases with scale if its value is less than the vertical correlation coefficient in cloud fraction between the two altitude levels. This occurs when the clouds are deeper than would be expected at random (i.e. for exponentially distributed cloud depths).
\end{abstract}

\section{Introduction}

Clouds tend to be represented in General Circulation Models (GCMs) as plane parallel and horizontally homogeneous, with the combined horizontal cloud fraction between clouds at different altitudes specified according to various overlap schemes (e.g. Smith, 1990; Tiedtke, 1993). These schemes are generally based on a combination of maximum and random overlap. In maximum overlap, the clouds are maximally overlapped in height, resulting in a minimum of interaction between clouds and downward radiation. Where clouds are randomly overlapped in height, the interaction with radiation is greater.

Taking advantage of the fact that clouds close together in altitude are likely maximally overlapped, and those significantly different in altitude are likely randomly overlapped, Hogan and Illingworth (2000) introduced a cloud overlap scheme that has since been widely taken up within GCMs. In this scheme, the mean combined cloud fraction between two altitude levels is taken to be a weighted average (with weight $\alpha$ ) of the mean values given by the maximum and random overlap assumptions respectively.

The value of $\alpha$ is generally taken to be a function of the height separation $(\Delta z)$ between the two altitudes, and is often found to have an inverse exponential dependence on $\Delta z$ (e.g. Hogan and Illingworth, 2000). The rate of fall is then determined by a cloud "decorrelation length" $L$ (i.e. $\alpha=e^{-\frac{\Delta z}{L}}$ ). Since this initial study of Hogan and Illingworth (2000), many others have investigated how $\alpha$ (and $L$ ) depend on horizontal scale (e.g. Mace and Benson-Troth, 2002; Oreopoulos and Khairoutdinov, 2003; Pincus et al., 2005; Willén et al., 2005; Barker, 2008a, b; Shonk and Hogan, 2010; Oreopoulos and Norris, 2011; Oreopoulos et al., 2012). Though a number of different definitions for $\alpha$ and methods for deriving $L$ have been used in such studies, they generally find that $\alpha$ (and, hence, $L$ ) increases with horizontal scale.

\section{The overlap parameter $\alpha$}

From the observed horizontal cloud fractions $c_{a}$ and $c_{b}$ at altitudes $a$ and $b$ (on a fixed scale), the horizontal cloud fractions $c_{\max }$ and $c_{\text {rand }}$ can be formed, under the maximum and 
random overlap schemes, as

$c_{\max }=\max \left(c_{a}, c_{b}\right)$

$c_{\text {rand }}=c_{a}+c_{b}-c_{a} c_{b}$.

From the definition as given by Hogan and Illingworth (2000) for $\alpha$, these are related to the combined horizontal cloud fraction $c_{t}$ (jointly covered by the clouds at both altitudes) by

$\overline{c_{t}}=\alpha \overline{c_{\max }}+(1-\alpha) \overline{c_{\text {rand }}}$,

where $\overline{c_{t}}, \overline{c_{\max }}$ and $\overline{c_{\text {rand }}}$ are the averages (over time) of $c_{t}$, $c_{\text {max }}$ and $c_{\text {rand }}$ respectively. For the idealised case given here, the averaging period is not important. However, most published work on cloud overlap is based on seasonal averages (e.g. Hogan and Illingworth, 2000; Oreopoulos and Norris, 2011).

Provided $\overline{c_{\max }}$ and $\overline{c_{\text {rand }}}$ are not equal to each other, which is unlikely (as this could only happen if the cloud cover fraction was always zero or one), Eq. (3) can be rearranged to give

$\alpha=\frac{\overline{c_{t}}-\overline{c_{\text {rand }}}}{\overline{c_{\max }}-\overline{c_{\text {rand }}}}$.

As pointed out in Pincus et al. (2005), this is only one way to define $\alpha$. Another method is to determine a set of values for $\alpha$ using Eq. (3), based on the individual (unaveraged) values of $c_{t}, c_{\max }$ and $c_{\text {rand }}$, and, from these, to find an average value for $\alpha$. However, this approach leads to data being discarded, as the values for $\alpha$ are not uniquely defined when either $c_{a}=0$ or $c_{b}=0$, potentially giving rise to truncated statistics. As the probability that $c_{a}=0$ or $c_{b}=0$ decreases with increasing grid size (e.g. Astin and Di Girolamo, 1999), it seems prudent, when considering the scale dependence, to use Eq. (4) to define $\alpha$ (in which no data are discarded).

\section{The horizontal scale dependence of $\alpha$}

To investigate the scale dependence of $\alpha$, we will consider what happens when two horizontally adjacent grid boxes, which we label $j$ and $j+1$ respectively, are combined to give a single larger grid box with double the area. In the following, there is no significance to $j$ or $j+1$, except as labels to distinguish the original two grid boxes. However, zonal and meridional anisotropies in real cloud regimes could make $\alpha$ directionally dependent. This would not affect the mathematics in this note, but should be kept in mind when applied to real data, if arbitrary pairs of adjacent grid boxes are combined. This could be handled by giving a direction to $j$ with, say, grid box $j+1$ being zonally (or meridionally) adjacent to grid box $j$. In either case, the cloud fractions $C_{a}$ and $C_{b}$ at the two altitudes ( $a$ and $b$ ) in the larger grid box are given by

$$
\left.\begin{array}{rl}
C_{a} & =\left(\frac{c_{a}(j)+c_{a}(j+1)}{2}\right) \\
C_{b} & =\left(\frac{c_{b}(j)+c_{b}(j+1)}{2}\right)
\end{array}\right\},
$$

where $c_{x}(y)$ is the cloud fraction in grid box $y$ at altitude $x$. Again, the cloud overlap $C_{\mathrm{MAX}}$ and $C_{\mathrm{RAND}}$ (on the larger scale) are formed, under the maximum and random overlap assumptions, by

$C_{\text {MAX }}=\max \left(C_{a}, C_{b}\right)$

$C_{\mathrm{RAND}}=C_{a}+C_{b}-C_{a} C_{b}$.

The combined cloud fraction, $C_{T}$, at a large scale is given by

$C_{T}=\frac{c_{t}(j)+c_{t}(j+1)}{2}$,

where $\mathrm{c}_{t}(y)$ is the combined cloud fraction in grid box $y$.

To continue, let $\alpha_{1}$ be the value of $\alpha$ at the original scale and $\alpha_{2}$ be the value of $\alpha$ when the two grid boxes are merged. As in Eq. (4), the value of $\alpha_{2}$ is given by

$\alpha_{2}=\frac{\overline{C_{T}}-\overline{C_{\mathrm{RAND}}}}{\overline{C_{\mathrm{MAX}}}-\overline{C_{\mathrm{RAND}}}}$,

where $\overline{C_{T}}, \overline{C_{\text {MAX }}}$ and $\overline{C_{\mathrm{RAND}}}$ are the time averages of $C_{T}$, $C_{\mathrm{MAX}}$ and $C_{\mathrm{RAND}}$ respectively.

Assuming that $\alpha$ depends only on scale (and the altitude between $a$ and $b$ ), then (using Eq. 3) Eq. (8) becomes

$\overline{C_{T}}=$

$\frac{\alpha_{1} \overline{c_{\max }(j)}+\left(1-\alpha_{1}\right) \overline{c_{\text {rand }}(j)}+\alpha_{1} \overline{c_{\max }(j+1)}+\left(1-\alpha_{1}\right) \overline{c_{\text {rand }}(j+1)}}{2}$

The averages in Eq. (10) are those for grid boxes $j$ and $j+1$ respectively. If $a$ and $b$ are fixed altitudes, then Eqs. (9) and (10) together imply that $\alpha_{2}=m \alpha_{1}+g$, where $m$ and $g$ are constants. This does not necessarily imply that a linear relationship between $\alpha_{1}$ and $\alpha_{2}$ will be observed, since data from different altitudes (likely having differing values of $m$ and $g$ ) may be combined in published studies.

For Eq. (10), we have implicitly assumed that $\alpha_{1}$ is the same for both grid boxes $j$ and $j+1$. To simplify the mathematics, in the following we will also assume that any average is the same, whether it is for grid box $j$ or for grid box $j+1$ (e.g. $\overline{c_{\max }(j)}=\overline{c_{\max }(j+1)}=\overline{c_{\max }}$ ). In Eq. (10), this is equivalent to dropping the $j$ and $j+1$ dependences, which together with Eq. (9) give

$\alpha_{2}=\frac{\overline{c_{\text {max }}}-\overline{c_{\text {rand }}}}{\overline{C_{\text {MAX }}}-\overline{C_{\text {RAND }}}} \alpha_{1}+\frac{\overline{c_{\text {rand }}}-\overline{C_{\text {RAND }}}}{\overline{C_{\text {MAX }}}-\overline{C_{\text {RAND }}}}$.

We can use Eq. (11) (or Eq. 10) to investigate the conditions under which $\alpha_{2}>\alpha_{1}$ (i.e. where $\alpha$ would increase with 
scale). As an example, consider the contrived case where the cloud cover varies between grid boxes, but is always the same at both heights $a$ and $b$ (i.e. $c_{a}(j)=c_{b}(j)$ and $c_{a}(j+1)=c_{b}(j+1)$, but $c_{a}(j)$ may not equal $\left.c_{a}(j+1)\right)$. This says nothing about the horizontal distribution of clouds at each height. However, this would seem most likely to be associated with particular cloud regimes, such as vertically deep convective clouds. For this case,

$$
\begin{aligned}
\overline{c_{\max }} & =\overline{\max \left(c_{a}(j), c_{b}(j)\right)} \\
& =\overline{\max \left(c_{a}(j), c_{a}(j)\right)}=\overline{c_{a}(j)},
\end{aligned}
$$

leading to

$\overline{c_{\max }}=\overline{c_{a}(j)}=\overline{c_{\mathrm{a}}}$.

Similarly, from Eq. (5), $C_{a}=C_{b}$ and $C_{\mathrm{MAX}}=$ $\max \left(C_{a}, C_{b}\right)=C_{a}$, giving

$\overline{C_{\mathrm{MAX}}}=\overline{C_{a}}=\overline{\left(\frac{c_{a}(j)+c_{a}(j+1)}{2}\right)}$.

As we are assuming that the averages are the same for both $j$ and $j+1$, Eq. (14) implies that $\overline{C_{\mathrm{MAX}}}=\overline{c_{a}}=\overline{c_{\max }}$ and $\alpha_{2}=m \alpha_{1}+(1-m)$. Hence, in this case, the value of $m$ is uniquely defined by the value of $\alpha_{2}$ when $\alpha_{1}$ equals zero (e.g. if $\alpha_{2}=0.2$ when $\alpha_{1}=0$, then $m=0.8$ and $\alpha_{2}=0.8 \alpha_{1}+0.2$ ).

It is instructive to consider this case further by studying the value of $m$ analytically. In this case, we can uniquely define a mean, $\mu$, and variance, $\sigma^{2}$, in cloud cover that is the same at both heights, i.e.

$$
\left.\begin{array}{c}
\mu=\overline{c_{a}(j)}=\overline{c_{b}(j)} \\
\sigma^{2}=\overline{c_{a}^{2}(j)}-\mu^{2}=\overline{c_{b}^{2}(j)}-\mu^{2}
\end{array}\right\} .
$$

In this case, $\overline{c_{\text {rand }}}$ is by definition (from Eq. 2)

$\overline{c_{\text {rand }}}=\overline{c_{a}(j)}+\overline{c_{b}(j)}-\overline{c_{a}(j) c_{b}(j)}=\mu+\mu-\overline{c_{a}^{2}(j)}$.

With Eq. (15), this gives

$\overline{c_{\text {rand }}}=2 \mu-\sigma^{2}-\mu^{2}$.

From Eqs. (7) and (14), the average $\overline{C_{\mathrm{RAND}}}$ is given by

$$
\begin{aligned}
\bar{C}_{\mathrm{RAND}} & =\bar{C}_{a}+\bar{C}_{b}-\overline{C_{a} C_{b}} \\
& =\bar{C}_{a}+\bar{C}_{a}-\overline{C_{a} C_{a}}=2 \mu-\bar{C}_{a}^{2} .
\end{aligned}
$$

This leads (from Eq. 5) to

$\overline{C_{\mathrm{RAND}}}=2 \mu-\overline{\left(\frac{c_{a}(j)+c_{a}(j+1)}{2}\right)^{2}}$.

Multiplying out gives

$$
\begin{aligned}
\overline{C_{\mathrm{RAND}}} & =2 \mu-\frac{1}{4} \overline{\left(c_{a}(j)\right)^{2}}-\frac{1}{4} \overline{\left(c_{a}(j+1)\right)^{2}} \\
& -\frac{1}{2} \overline{c_{a}(j) c_{a}(j+1)} .
\end{aligned}
$$

Again, assuming that the averages are the same in both grid boxes, the mean, $\mu$, and variance, $\sigma$, in cloud cover are the same for both grid boxes $j$ and $j+1$, and retain their definitions as given in Eq. (15). In this case, the labels $j$ and $j+1$ are redundant in the second and third terms on the RHS of Eq. (20), and can be dropped to give

$\overline{C_{\mathrm{RAND}}}=2 \mu-\frac{1}{4} \overline{c_{a}^{2}}-\frac{1}{4} \overline{c_{a}^{2}}-\frac{1}{2} \overline{c_{a}(j) c_{a}(j+1)}$.

From Eq. (15), this reduces to

$$
\overline{C_{\mathrm{RAND}}}=2 \mu-\frac{1}{2}\left(\sigma^{2}+\mu^{2}\right)-\frac{1}{2} \overline{c_{a}(j) c_{a}(j+1)} .
$$

By definition, the co-variance of $c_{a}(j)$ and $c_{a}(j+1)$ is given by

$\operatorname{Cov}\left(c_{a}(j), c_{a}(j+1)\right)=\overline{c_{a}(j) c_{a}(j+1)}-\mu^{2}$.

Similarly, by definition, the (horizontal) cross-correlation coefficient, $R$, in cloud cover between the adjacent (smaller) grid boxes is given by

$$
\begin{aligned}
R & =\frac{\operatorname{Cov}\left(c_{a}(j), c_{a}(j+1)\right)}{\sqrt{\operatorname{Var}\left(c_{a}(j)\right)} \sqrt{\operatorname{Var}\left(c_{a}(j+1)\right)}} \\
& =\frac{\operatorname{Cov}\left(c_{a}(j), c_{a}(j+1)\right)}{\sigma^{2}} .
\end{aligned}
$$

Equations (22), (23) and (24) together give

$$
\begin{aligned}
\overline{C_{\mathrm{RAND}}} & =2 \mu-\frac{1}{2}\left(\sigma^{2}+\mu^{2}\right)-\frac{R}{2} \sigma^{2} \\
& -\frac{1}{2} \mu^{2}=2 \mu-\frac{1}{2}(1+R) \sigma^{2}-\mu^{2} .
\end{aligned}
$$

Putting these into Eq. (11) gives

$$
m=\frac{\overline{c_{\text {max }}}-\overline{c_{\text {rand }}}}{\overline{C_{\mathrm{MAX}}}-\overline{C_{\mathrm{RAND}}}}=\frac{\mu-\sigma^{2}-\mu^{2}}{\mu-\frac{1}{2}(1+R) \sigma^{2}-\mu^{2}} .
$$

As an example, if the cloud fraction can be modelled as a $\operatorname{Beta}(p, q)$ distribution (e.g. Falls, 1974; Tompkins, 2002), then

$$
\begin{aligned}
m & =\frac{2(p+q)}{2(p+q)+(1-R)} \\
\alpha_{2} & =\frac{2(p+q)}{2(p+q)+(1-R)} \alpha_{1}+\frac{(1-R)}{2(p+q)+(1-R)} .
\end{aligned}
$$

In the simplest case, where the cloud fraction in each grid box is uniformly or $\operatorname{Beta}(1,1)$ distributed (e.g. LeTreut and Li, 1991), Eq. (28) gives

$\alpha_{2}=\frac{4}{5-R} \alpha_{1}+\frac{1-R}{5-R}$.

(Thus, where $R=0$, then $\alpha_{2}=0.8 \alpha_{1}+0.2$ ). Hence, in this contrived case (where the cloud cover is the same at both 
heights), $\alpha$ will always increase with scale (i.e. $\alpha_{2}>\alpha_{1}$ ) provided that the horizontal correlation coefficient, $R$, in the cloud fraction between adjacent grid boxes is positive and less than 1 .

Trivially, when $R=1$, there is no scale dependence on $\alpha$ (as $m=1$ ). However, as $R$ decreases to zero, the degree of the scale dependence increases, and maximises where $R=0$. This is displayed in Fig. 1, which shows the relationship between $\alpha_{1}$ and $\alpha_{2}$ for a range of values for $R$ in the case where the cloud fraction in the adjacent grid boxes is assumed to be uniformly distributed. The scale dependence is strongest when $R=0$, in which $\alpha_{2}=0.8 \alpha_{1}+0.2$.

So far, we have looked at the scale dependence where the cloud fraction varies from grid box to grid box, but does not vary with altitude. This implies that the vertical correlation between the cloud fractions at the two altitudes is $\rho=1$. Let us now consider what happens when $\overline{c_{a}}=\overline{c_{b}}$, but $c_{a}(j)$ need not equal $c_{b}(j)$ (i.e. $\rho \neq 1$ ). For illustration, and to simplify the mathematics, we will take the extreme case where $R=0$ and assume that the cloud cover fractions at heights $a$ and $b$ are correlated uniform distributions, with a (vertical) correlation coefficient $\rho$. This implies that the mean cloud fraction at each height is $\mu=\frac{1}{2}$.

From Clark (1961) or Nadarajah and Kotz (2008) for example, the mean $\left(\overline{c_{\max }}\right)$ of the maximum of two correlated normally distributed random variables with mean $\mu=\frac{1}{2}$, standard deviation $\sigma$ and correlation coefficient $\rho$ is given by

$\overline{c_{\max }}=\frac{1}{2}+k(1-\rho)^{1 / 2}$,

where $k=\sigma^{2} \pi^{-0.5}$.

We could not find a reference for the mean of the maximum of two correlated uniform random variables, so we will use Eq. (30), with $k$ chosen to give the correct answer for $\overline{c_{\max }}$ when $\rho=0$. (Equation 30 will always give the correct answer when $\rho=1$.) We will comment later on the accuracy of this assumption.

If $c_{a}$ and $c_{b}$ are independent uniformly distributed random variables, then $\rho=0$ and $c_{\max }$ follows a $\operatorname{Beta}(2,1)$ distribution, which has mean $\overline{c_{\max }}=\frac{2}{2+1}=\frac{2}{3}$. Hence, Eq. (30) gives the correct value for $\bar{c}_{\text {max }}$ if $k=\frac{1}{6}$. This leads to

$\overline{c_{\max }} \cong \frac{1}{2}+\frac{1}{6}(1-\rho)^{1 / 2}$.

Also, when $c_{a}$ and $c_{b}$ are independent uniformly distributed random variables, their average $C_{a}$ has the standard symmetric triangular distribution, as does $C_{b}$. Hence, $\overline{C_{\mathrm{MAX}}}$ is the mean of the maximum of two independent triangularly distributed random variables. In this case, $\overline{C_{\mathrm{MAX}}}=\frac{37}{60}$, and Eq. (30) gives the correct value if $k=\frac{7}{60}$. This leads to

$\overline{C_{\mathrm{MAX}}} \cong \frac{1}{2}+\frac{7}{60}(1-\rho)^{1 / 2}$.
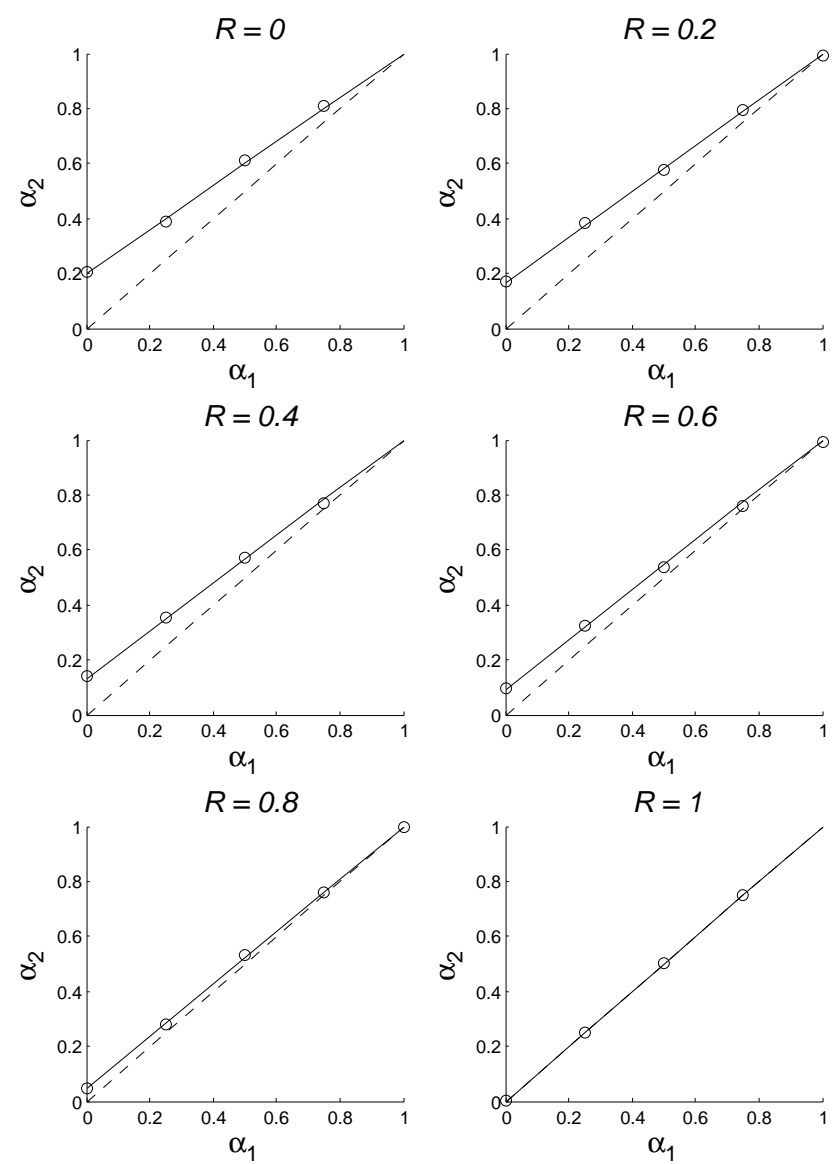

Figure 1. The dependence of $\alpha_{2}$ on $\alpha_{1}$ for cloud fractions (in adjacent grid boxes) that are uniformly distributed, where the vertical correlation coefficient in cloud cover $\rho=1$ and the horizontal correlation coefficient in cloud cover is $R$ (solid line). The dashed line is where there would be no scale dependence to $\alpha$ (i.e. $\alpha_{2}=\alpha_{1}$ ). The circles are values given by simulation.

In a similar way to $R$, the vertical correlation coefficient $\rho$ is defined as

$$
\begin{aligned}
\rho & =\frac{\operatorname{Cov}\left(c_{a}(j), c_{b}(j)\right)}{\sqrt{\operatorname{Var}\left(c_{a}(j)\right)} \sqrt{\operatorname{Var}\left(c_{b}(j)\right)}} \\
& =\frac{\operatorname{Cov}\left(c_{a}(j), c_{b}(j)\right)}{\sigma^{2}}=\frac{\overline{c_{a} c_{b}}-\mu^{2}}{\sigma^{2}} .
\end{aligned}
$$

Based on Eq. (2), Eq. (33) gives

$$
\overline{c_{\text {rand }}}=\overline{c_{a}}+\overline{c_{b}}-\overline{c_{a} c_{b}}=2 \mu-\mu^{2}-\sigma^{2} \rho
$$

(This is identical to Eq. 17 when $\rho=1$.) For a uniform distribution $\sigma^{2}=\frac{1}{12}$, giving

$\overline{c_{\text {rand }}}=\frac{3}{4}-\frac{1}{12} \rho$. 
Similarly,

$$
\begin{aligned}
& \bar{C}_{\mathrm{RAND}}=\overline{C_{a}}+\overline{C_{b}}-\overline{C_{a} C_{b}} \\
& =2 \mu-\frac{\overline{\left(c_{a}(j)+c_{a}(j+1)\right)}}{2} \frac{\left(c_{b}(j)+c_{b}(j+1)\right)}{2} .
\end{aligned}
$$

Multiplying out gives

$$
\begin{aligned}
\overline{C_{\mathrm{RAND}}} & =2 \mu-\left(\overline{\frac{c_{a}(j) c_{b}(j)}{4}}+\frac{\overline{c_{a}(j) c_{b}(j+1)}}{4}\right. \\
& \left.+\frac{\overline{c_{a}(j+1) c_{b}(j)}}{4}+\frac{\overline{c_{a}(j+1) c_{b}(j+1)}}{4}\right) .
\end{aligned}
$$

As we are only considering the case where $R=0$ (i.e. no horizontal correlation), this simplifies Eq. (23) to

$$
\begin{aligned}
\overline{C_{\mathrm{RAND}}} & =2 \mu-\frac{\overline{c_{a}(j) c_{b}(j)}}{4}-\frac{\mu^{2}}{4} \\
& -\frac{\mu^{2}}{4}-\frac{\overline{c_{a}(j+1) c_{b}(j+1)}}{4} .
\end{aligned}
$$

As the averages are the same for both $j$ and $j+1$,

$$
\begin{aligned}
\overline{C_{\mathrm{RAND}}} & =2 \mu-\frac{1}{2} \mu^{2}-\frac{1}{2} \overline{c_{a} c_{b}} \\
& =2 \mu-\frac{1}{2} \mu^{2}-\frac{1}{2}\left(\mu^{2}+\sigma^{2} \rho\right)
\end{aligned}
$$

$\overline{C_{\mathrm{RAND}}}=2 \mu-\frac{1}{2} \mu^{2}-\frac{1}{2} \overline{c_{a} c_{b}}=2 \mu-\mu^{2}-\frac{1}{2} \sigma^{2} \rho$.

$\overline{C_{\text {RAND }}}=\frac{3}{4}-\frac{1}{24} \rho$

Putting the above values into Eq. (11) gives

$$
\begin{aligned}
\alpha_{2} & \cong \alpha_{1}\left(\frac{30-10 \rho-20(1-\rho)^{1 / 2}}{30-5 \rho-14(1-\rho)^{1 / 2}}\right) \\
& +\left(\frac{5 \rho}{30-5 \rho-14(1-\rho)^{1 / 2}}\right) .
\end{aligned}
$$

Though this is an approximate result, the simulated values given in Fig. 2 show that Eq. (42) can be taken as exact for all values of $\rho$. Thus, if $\rho=0$ (i.e. the cloud cover at both altitudes is uncorrelated), $\alpha_{2}=\frac{5}{8} \alpha_{1}$, and so $\alpha$ will always decrease with scale (i.e. $\alpha_{2}<\alpha_{1}$ ), except where $\alpha_{1}=0$.

It seems likely, given the linear relationship between the values of $\alpha$ on the two scales, that for every value of $\rho$, there will be a unique value for $\alpha$ that does not change with scale, being the point of intersection with the $\alpha_{1}=\alpha_{2}$ line. This is illustrated in Fig. 2, where the relationship between $\alpha_{1}$ and $\alpha_{2}$ is displayed for a range of values for $\rho$ (all with $R=0$ ). From Fig. 2 , this value seems to be where $\alpha_{1}=\alpha_{2} \approx \rho$. Also, where $\alpha_{1}>\rho$, then $\alpha$ will decrease with scale, and where $\alpha_{1}<\rho$, then $\alpha$ will increase with scale.
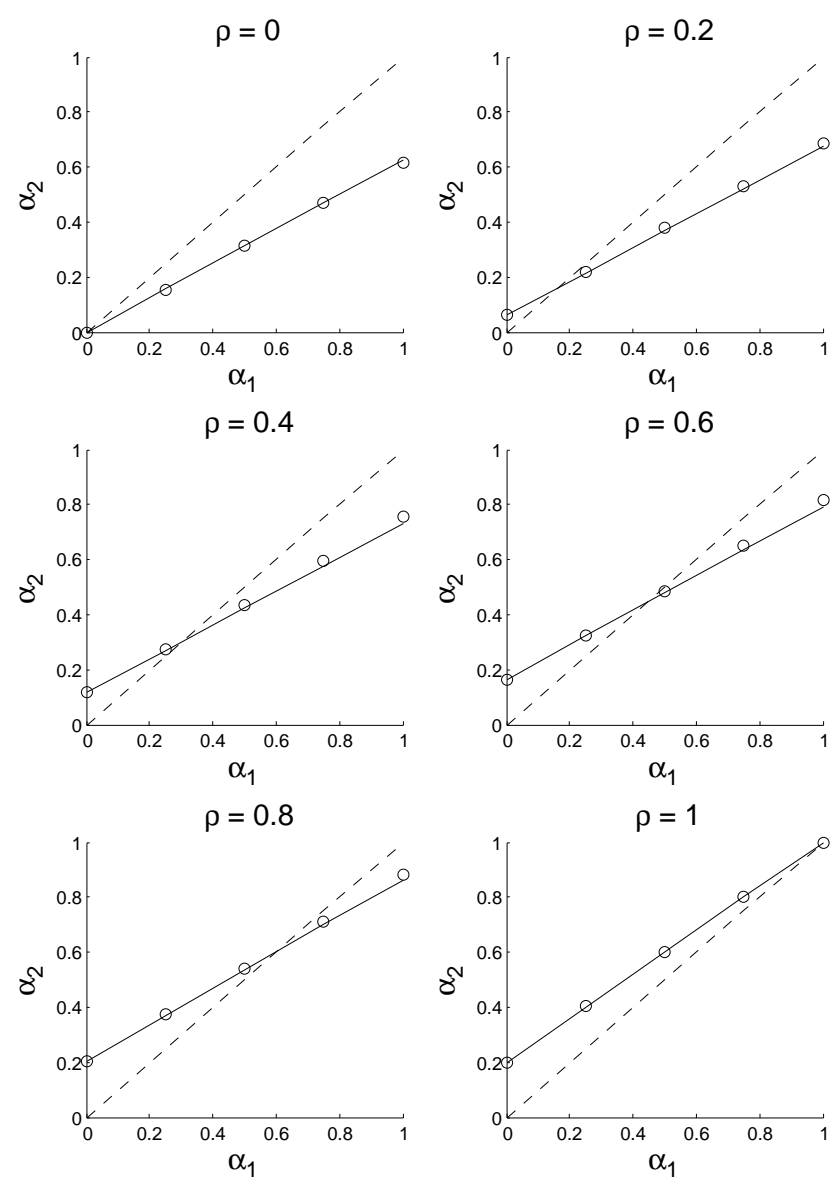

Figure 2. The dependence of $\alpha_{2}$ on $\alpha_{1}$ for cloud fractions that are uniformly distributed (solid line), where the horizontal correlation coefficient in cloud cover is $R=0$, and the vertical correlation coefficient in cloud cover is $\rho$. The dashed line is where there would be no scale dependence on $\alpha$ (i.e. $\alpha_{2}=\alpha_{1}$ ). The circles are values from simulation.

\section{Conclusions}

Based on the definition of $\alpha$ and the scale invariance of the combined cloud fraction, if $\alpha$ depends only on scale, then the value of alpha, $\alpha_{2}$, on one scale is linearly related to the value of alpha, $\alpha_{1}$, on the other scale (i.e. $\alpha_{2}=m \alpha_{1}+g$ ), provided that the two altitudes are fixed. The values of $m$ and $g$ depend on a number of parameters, including the mean, $\mu$, and variance, $\sigma^{2}$, in cloud fraction at each altitude. However, the most important parameters are the horizontal correlation coefficient, $R$, between the cloud fractions in adjacent grid boxes (at a given altitude) and the vertical correlation coefficient, $\rho$, between the cloud fractions at the two altitudes.

If $R, \rho, \mu$ and $\sigma^{2}$ are found from real cloud data, then this note allows the value of $\alpha_{2}$ to be calculated from $\alpha_{1}$ directly. Being horizontal cloud properties, $R, \mu$ and $\sigma^{2}$ can be found directly from the passive or active remote sensing of clouds. However, $\rho$ would require knowledge of 
cloud vertical structure, which could come from active remote sensing (e.g. as in Kato et al., 2010, from CloudSat and CALIPSO data).

Dependent on the relative values of $\alpha$ and $\rho$, it is possible for $\alpha$ to increase, decrease or stay the same with increasing scale. However, the strength of the dependence is controlled by $R$. Published results tend to obscure the linear relationship between $\alpha_{2}$ and $\alpha_{1}$ by plotting them together on the same graph against height separation, rather than against one another (e.g. Oreopoulos and Norris, 2011). This also combines data from differing pairs of altitudes ( $a$ and $b$ ) together, where each pair could have a different linear relationship. However, our results indicate that an "on average" increase in $\alpha$ with scale implies that, on average, $\alpha$ must generally be smaller than $\rho$.

In Astin and Di Girolamo (2006), we showed that on average, $\alpha \approx \rho$ when cloud depths follow an exponential distribution. Hence, we conclude that the published increase in $\alpha$ with scale is a consequence of clouds being generally deeper than would be expected at random (i.e. in a random Markov field).

Also, the scale dependence disappears when $R=1$, and is strongest when $R=0$. Hence, an increase in $\alpha$ with scale implies that $R$ must be positive and less than 1. Based on published data on $\alpha$, or directly from cloud data, it is possible to determine $R$ if there is enough data to determine $\rho$, $\mu$ and $\sigma^{2}$. As an illustration, Fig. 1 of Oreopoulos and Norris (2011) gives $\alpha_{1} \approx 0$ (at $75 \mathrm{~km}$ scale) and $\alpha_{2} \approx 0.04$ (at $150 \mathrm{~km}$ scale) for an altitude separation of $10 \mathrm{~km}$ when averaged over June, July and August. Based on this note, this would indicate that if $\rho=0$, then $R$ has a maximum value of 0.8 (our Fig. 1). However, $R$ could equal zero, provided that $\rho \geq 0.2$ (our Fig. 2). As $\rho$ is likely to be close in value to $\alpha_{1}$, this would seem to imply that $R$ is closer to 0 than 0.8 . This is a wide range for $R$, but could be made narrower if $\rho$ is known.

Acknowledgements. We would like to thank the anonymous reviewers whose comments have made this a better and more readable paper. We would also like to thank Lazaros Oreopoulos and Adrian Tompkins for their helpful comments during the review process.

Edited by: J. Quaas

\section{References}

Astin, I. and Di Girolamo, L.: A general formalism for the distribution of the total length of a geophysical parameter along a finite transect, IEEE T. Geosci. Remote Sens., 37, 508-512, 1999.

Astin, I. and Di Girolamo, L.: The relationship between alpha and the cross-correlation of cloud fraction, Q. J. Roy. Meteorol. Soc., 132, 2475-2478, doi:10.1256/qj.05.209, 2006.

Barker, H. W.: Overlap of fractional cloud for radiation calculations in GCMs: A global analysis using CloudSat and CALIPSO data,
J. Geophys. Res., 113, D00A01, doi:10.1029/2007JD009677, 2008a.

Barker, H. W.: Representing cloud overlap with an effective decorrelation length: An assessment using CloudSat and CALIPSO data, J. Geophys. Res., 113, D24205, doi:10.1029/2008JD010391, 2008b.

Clark, C. E.: The greatest of a finite set of random variables, Operations Res., 9, 145-162, 1961.

Falls, L. W.: The beta distribution: A statistical model for world cloud cover, J. Geophys. Res., 79, 1261-1264, doi:10.1029/JC079i009p01261, 1974.

Hogan, R. J. and Illingworth, A. J.: Deriving cloud overlap statistics from radar, Q. J. Roy. Meteorol. Soc., 126, 2903-2909, 2000.

Kato, S., Sun-Mack, S., Miller, W. F., Rose, F. G., Chen, Y., Minnis, P., and Wielicki, B. A.: Relationships among cloud occurrence frequency, overlap, and effective thickness derived from CALIPSO and CloudSat merged cloud vertical profiles, J. Geophys. Res., 115, D00H28, doi:10.1029/2009JD012277, 2010.

LeTreut, H. and Li, Z. X.: Sensitivity of an atmospheric general circulation model to prescribed SST changes: Feedback effects associated with the simulation of cloud optical properties, Clim. Dynam., 5, 175-187, 1991.

Mace, G. G. and Benson-Troth, S.: Cloud-layer characteristics derived from long-term cloud radar data, J. Climate, 15, 25052515, 2002.

Nadarajah, S. and Kotz, S.: Exact distribution of the max/min of two Gaussian random variables, IEEE Trans. Very Large Scale Integr. Syst., 16, 210-212, 2008.

Oreopoulos, L. and Khairoutdinov, M.: Overlap properties of clouds generated by a cloud-resolving model, J. Geophys. Res., 108, 4479-4488, 2003.

Oreopoulos, L. and Norris, P. M.: An analysis of cloud overlap at a midlatitude atmospheric observation facility, Atmos. Chem. Phys., 11, 5557-5567, doi:10.5194/acp-11-5557-2011, 2011.

Oreopoulos, L., Lee, D., Sud, Y. C., and Suarez, M. J.: Radiative impacts of cloud heterogeneity and overlap in an atmospheric General Circulation Model, Atmos. Chem. Phys., 12, 9097-9111, doi:10.5194/acp-12-9097-2012, 2012.

Pincus, R., Hannay, C., Klein, S. A., Xu, K.-M., and Hemler, R.: Overlap assumptions for assumed probability distribution function cloud schemes in large-scale models, J. Geophys. Res., 110, D15S09, doi:10.1029/2004jd005100, 2005.

Shonk, J. K. P. and Hogan, R. J.: Effect of improving representation of horizontal and vertical cloud structure on the earth's global radiation budget. Part II: the global effects, Q. J. Roy. Meteorol. Soc., 136, 1205-1215, doi:10.1002/qj.646, 2010.

Smith, R. N. B.: A scheme for predicting layer clouds and their water content in a general circulation model, Q. J. Roy. Meteorol. Soc., 116, 435-460, 1990.

Tiedtke, M.: Representation of clouds in large-scale models, Mon. Weather Rev., 121, 3040-3061, 1993

Tompkins, A. M.: A prognostic parameterization for the subgridscale variability of water vapor and clouds in large-scale models and its use to diagnose cloud cover, J. Atmos. Sci., 59, 19171942, 2002.

Willén, U., Crewell, S., Baltink, H. K., and Sievers, O.: Assessing model predicted vertical cloud structure and cloud overlap with radar and lidar ceilometer observations for the Baltex Bridge Campaign of CLIWA-NET, Atmos. Res., 75, 227-255, 2005. 